\title{
The Research of Velocity Factors Sensitivity in Turbine Stages under Off-design Conditions
}

\author{
Junxu Jiang ${ }^{1}$, Jianqun $\mathrm{Xu}^{1 *}$, Lili Zhou ${ }^{1}$, Tianyang Xing ${ }^{1}$, Hanyu Wang ${ }^{1}$ \\ ${ }^{1}$ Southeast University, Department of Energy and Environment, Sipailou 2, Nanjing, China
}

\begin{abstract}
Velocity factors are important parameters to determine the efficiency of turbine stages, and it is necessary to systematically study the effect of decline in velocity factors on stages efficiency under offdesign conditions. In this paper the definition of the sensitivity of the velocity factors is proposed and effect of each parameter on the sensitivity is analysed. A simplified off-design model was also established to study the change of sensitivity of a governing-stage and pressure stage under the varying mass flow conditions. The results show that for the calculated turbine, the nozzle sensitivity of the governing stage is always greater than the blade for off-design conditions, and the efficiency of the nozzle in the governing stage should be emphasized. While the sensitivity of the pressure stage is related to the flow rate, it is more meaningful to improve the blade velocity factors in large mass flow conditions and it is reverse for low mass flow conditions. These findings will be meaningful in the optimization of turbine operation and design
\end{abstract}

\section{Introduction}

Recent years, with the rapid development of renewable energy, the structure of the energy production has a tremendous change. Due to the randomness of renewable energy, the stability of national grid is dramatic decline. In order to ensure the safety of the grid, the steam turbine in the power plant must to work in low flow rate conditions which make the process in the stage changes greatly. Therefore, the research of the stage process in off-design condition is a prerequisite for optimizing the turbine design and operation in this situation.

The velocity factors of the stage, which corresponds to the enthalpy loss coefficient ${ }^{[1]}$, is the ratio of the actual velocity to the isentropic velocity at blade or nozzle outlet, and can characterize the losses in the nozzle or blade due to friction and other factors. It is influenced by many manufacturing and operating parameters ${ }^{[2]}$, and many literature $e^{[3]-[4]}$ have studied the effect caused by the variation of the velocity factors. For instance, the efficiency loss caused by the reduction of the nozzle factors is approximately four times compared to the blade factors reduction ${ }^{[5]}$. However, the current studies are often limited to the design conditions and lack the research under off-design conditions.

Therefore, this paper addresses the limitations of the above literature in the research of velocity factors losses by (1) proposing the definition of sensitivity of velocity factors and theoretically deriving the equations for the sensitivity and the sensitivity ratio before an influence parameters analysis of velocity factors sensitivity .(2) A simplified off-design model was developed for calculating the assume velocity ratio and reaction degree of the stage, which is used for sensitivity analysis under low flow rate conditions, and validated it with available data.(3)At last, the results of the sensitivity variation after the mass flow rate change were calculated and discussed.

\section{Theoretical analysis of velocity factors sensitivity}

\subsection{The Definition of Velocity Factor Sensitivity}

The adiabatic turbine stages model of the axial turbine is used in this paper, the performance of stages can be represented in an enthalpy-entropy diagram in Fig.1.

The velocity factors of the nozzle and blade can be defined as:

$$
\varphi=\frac{c_{1}}{c_{1 t}}, \psi=\frac{w_{2}}{w_{2 t}}
$$

Where $\varphi, \psi$ means nozzle and blade velocity factors respectively. $c_{1}, c_{1 t}$ means Actual and ideal velocities of nozzle exit. And $w_{2}, w_{2 t}$ means actual and ideal relative velocities at the outlet of the blade

* Corresponding author: qlj1062@163.com 


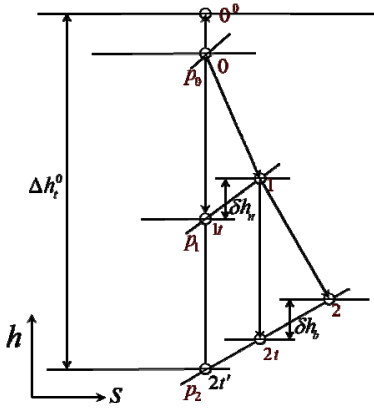

Figure 1. Enthalpy-Entropy Diagram of Stages.

According to the concept of velocity factor sensitivity as the effect of a variation in velocity factors on the stage efficiency, the sensitivity can be defined as below:

Sensitivity $=\frac{\text { efficiency loss after } 1 \% \text { absolute decline in nozzle/blade factors }}{\text { the wheel efficiency before factors decline }}$ which can be noted as :

$$
s_{n}=\frac{\Delta \eta_{u}^{n}}{\eta_{u}^{0}}, \quad s_{b}=\frac{\Delta \eta_{u}^{b}}{\eta^{0}{ }_{u}}
$$

Where $s_{n}, s_{b}$ mean the sensitivity of nozzle and blade respectively. $\Delta \eta_{u}^{n}, \Delta \eta_{u}^{b}, \eta^{0}{ }_{u}$ mean the efficiency loss due to nozzle and balde factors decline and the wheel efficiency before the factors change.

Finally, the ratio of nozzle and blade sensitivity is defined as the sensitivity ratio, denoted as $S$, and the equation is shown as below:

$$
S=\frac{s_{n}}{s_{b}}
$$

\subsection{The Derivation of Sensitivity model}

According to the above model assumptions, it is not difficult to obtain the equation of the wheel efficiency in a normal stage as below. And the specific definition of each symbol can be found in literature [3].

$$
\eta_{u}=\frac{2 x_{a}\left(\varphi \sqrt{1-\Omega_{m}} \cos \alpha_{1}+\psi \Phi \cos \beta_{2}-x_{a}\right)}{1-\mu_{1}\left[(\Phi \psi)^{2}+x_{a}^{2}-2 \Phi x_{a} \psi \cos \beta_{2}\right]}
$$

Replaces the numerator, which means the wheel efficiency without considering the exit velocity utilazation, with the symbol $A$. And The effect of the original exit velocity utilization (denominator) is changed to a reciprocal correction $\frac{1}{C}$.

Therefore the equations (2) and (3) can be simplified by the above derivation to the below form.

$$
\begin{gathered}
s_{n}=1-\frac{A^{n}}{A} \times \frac{C}{C^{n}}, s_{b}=1-\frac{A^{b}}{A} \times \frac{C}{C^{b}} \\
S=\frac{\frac{A}{C}-\frac{A^{n}}{C^{n}}}{\frac{A}{C}-\frac{A^{b}}{C^{b}}}=\frac{1-\frac{A^{n}}{A} \times \frac{C}{C^{n}}}{1-\frac{A^{b}}{A} \times \frac{C}{C^{b}}}
\end{gathered}
$$

where the superscripts $n$ and $b$ indicate the state when the absolute velocity factor of the nozzle and blade is reduced by $1 \%$, respectively.

The above equation can also be expressed in the form as :

$$
\left[s_{n}, s_{b}, S\right]=F\left(x_{a}, \Omega_{m}, \alpha_{1}, \beta_{2}, \varphi, \psi\right)
$$

That means the sensitivity is related to the parameters like assume velocty ratio,the reaction degree, the structure and the velocity factor of a stage.

\subsection{Factor Analysis of Sensitivity}

In order to study the effect of each parameter on the sensitivity, the incremental form of the sensitivity for each parameter, which is shown as blow, is considered.

$$
\frac{\partial\left[s_{n}, s_{b}\right]}{\partial v}=\frac{\partial F\left(x_{a}, \Omega_{m}, \alpha_{1}, \beta_{2}, \varphi, \psi\right)}{\partial v} \Delta v \quad\left(v=x_{a}, \Omega_{m}, \varphi, \psi\right)
$$

The sensitivity function is a basic equation composed of continuous functions, so it is not difficult to prove the continuity of its partial derivatives. The summation of increment is used to obtain the amount of change in sensitivity when each parameter is changed. And this result can be used to obtain the main factors that influence the sensitivity in the case of off-design conditons.

Then a real impulse stage and reaction stage are calculated and analysed. In the process of calculating, the reaction degree $\Omega_{m}$, assume velocity ratio $x_{a}$, nozzle and blade factors $\varphi, \psi$ is taken account. Considering the actual process, the impulse stage parameters change interval is larger and the trend is the same, take its parameter variation interval is $-80 \% \sim 0 \%$ (blade and nozzle factors are $-10 \% \sim 0$ ), while the reaction stage change interval is smaller and the trend is different, its parameter variation interval is $-10 \% \sim 5 \%$. The calculated results are shown in Figures 2 and 3 below.

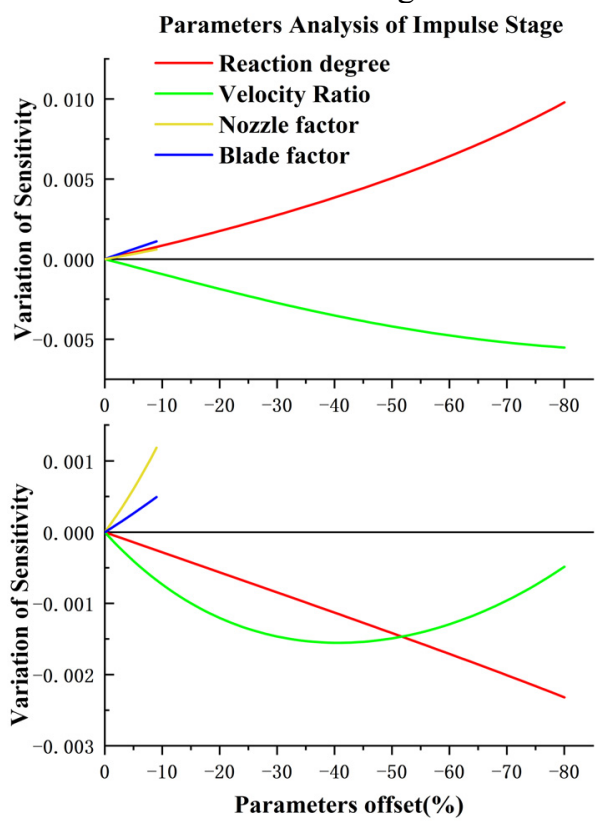

Figure 2. Parameters Analysis of Sensitivity in Impulse stages (the top is nozzle sensitivity and the bottom is blade).

As it is depicted in Figure 2, for the impulse stage, the nozzle and blade sensitivity, except the speed ratio, is basically a linear trend for each parameter in the interval. In the whole interval, the reaction degree and velocity ratio have significant influence on the two sensitivity. And for the impulse stage because the reaction degree is 
relatively small so the size of the change in nozzle sensitivity is significantly larger than the blade sensitivity.

In the parameters analysis of blade sensitivity, sensitivity changes with the velocity ratio is in a quadratic function relationship. This is because the actual impulse stage selected a larger velocity in design parameters. When the velocity ratio decreases, the wheel efficiency increases first and then decreases. And because the blade loss in the impulse stage with the velocity ratio changes are not obvious, so the blade sensitivity has an opposite trend of efficiency changes with the velocity ratio.

Parameters Analysis of Reaction Stage
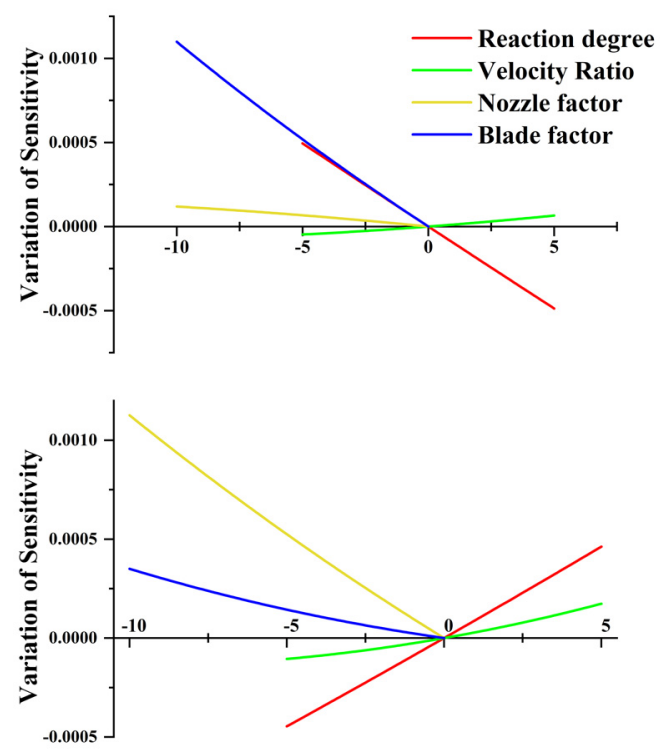

Parameters offset( $\%)$

Figure 3. Parameters Analysis of Sensitivity in reaction stages (the top is nozzle sensitivity and the bottom is blade).

As shown in the Figure 3, the sensitivity change in the reaction stage for each parameter is basically the same. There is no more major influencing factors, because the parameters such as reaction degree has an unconspicuous variation due to the condition change. Therefore, sensitivities like other factors would have a stable trend under off-design conditions.

At last, as the tow figure shows that the reaction degree is the main factor affecting the sensitivity. When the reaction degree increases the blade sensitivity increases while the nozzle sensitivity decreases. This is because the reaction degree determines the enthalpy drop proportion of the stage. For example, an increase in reaction degree will lead to an argument in the enthalpy drop of blade, and the loss proportion of the loss will relatively raise. Therefore, the sensitivity will change as the mentioned above.

\section{The Sensitivity Analysis under Off- design Conditions}

\subsection{The Simplified Model under Off-design Conditions}

In order to analyse the variation of sensitivity of a general stage under off-design conditions, it is necessary to obtain the velocity ratio and reaction degree of the stage in this situation. A simplified model was established to acquire the two parameters in low flow rate conditions for the condensing steam turbine stages in the non-critical situation. For the governing stage using the nozzle governing, the fast algorithm of the literature [6] is used and the pressure ratio of its stage is considered to vary linearly with the flow rate, so its enthalpy drop and velocity ratio are easily found. The general pressure stage can be established by the Freugel's equation like below.

$$
\frac{G}{G_{0}}=\sqrt{\frac{p_{1}^{2}-p_{g}^{2}}{p_{01}^{2}-p_{0 g}^{2}}}=\sqrt{\frac{p_{2}^{2}-p_{g}^{2}}{p_{02}^{2}-p_{0 g}^{2}}}
$$

Where the subscripts 1,2 ,g mean the state before the stage, the state after the stage and the garge pressure in condenser, and 0 means the original working condition. It can be deduced from the above that the pressrue ratio after conditions change is like below :

$$
\frac{p_{2}}{p_{1}}=\sqrt{\frac{p_{g}^{2}+\left(p_{01}^{2}-p_{0 g}^{2}\right)\left(\frac{G_{0}}{G}\right)^{2}}{p_{g}^{2}+\left(p_{02}^{2}-p_{0 g}^{2}\right)\left(\frac{G_{0}}{G}\right)^{2}}}
$$

After obtaining pressure ratio, the ideal enthalpy drop ratio can be obtained by substituting ideal enthalpy drop equation to above.

$$
\frac{\Delta h_{t}}{\Delta h_{t 0}}=\frac{T}{T_{0}} \frac{1}{1-\left(\frac{p_{02}}{p_{01}}\right)^{\frac{\kappa-1}{\kappa}}}\left\{1-\left[\sqrt{\frac{p_{g}^{2}+\left(p_{01}^{2}-p_{0 g}^{2}\right)\left(\frac{G_{0}}{G}\right)^{2}}{p_{g}^{2}+\left(p_{02}^{2}-p_{0 g}^{2}\right)\left(\frac{G_{0}}{G}\right)^{2}}}\right]^{\frac{\kappa-1}{\kappa}}\right\}
$$

Where $T$ means temperature and $\kappa$ is the adiabatic index of superheated steam, assuming :

$$
\begin{gathered}
B=\frac{T}{T_{0}\left[1-\left(\frac{p_{02}}{p_{01}}\right)^{\frac{\kappa-1}{\kappa}}\right.} \overline{[}\left[\sqrt{\frac{p_{g}^{2}+\left(p_{01}^{2}-p_{0 g}^{2}\right)\left(\frac{G_{0}}{G}\right)^{2}}{p_{g}^{2}+\left(p_{02}^{2}-p_{0 g}^{2}\right)\left(\frac{G_{0}}{G}\right)^{2}}}\right]^{\frac{\kappa-1}{\kappa}} \\
M=1-
\end{gathered}
$$

Then, by the definition of assume velocity ratio, the equation under the off-design conditions can be expressed like below.

$$
x_{a}=\frac{u_{1}}{c_{a}}=\frac{u_{1}}{\sqrt{2 \Delta h_{t}}}=\frac{u_{1}}{\sqrt{2 B M \Delta h_{t 0}}}=\frac{1}{\sqrt{B M}} x_{a 0}
$$

Meanwhile, according to the literature [7], the reaction degree of the stage can be expressed by an implicit function containing the area ratio of the blade and nozzle, the pressure ratio, and the velocity ratio, as shown in the following equation. 


$$
\Omega_{m}=F\left(f, x_{a}, \varepsilon\right)
$$

In order to prove the accuracy of the above model, the calculated results were verified with the real date of a low pressure stage in a 300MW unit and a impulsive governing stage provided in the literature [8]. The results are shown in Fig. 4.
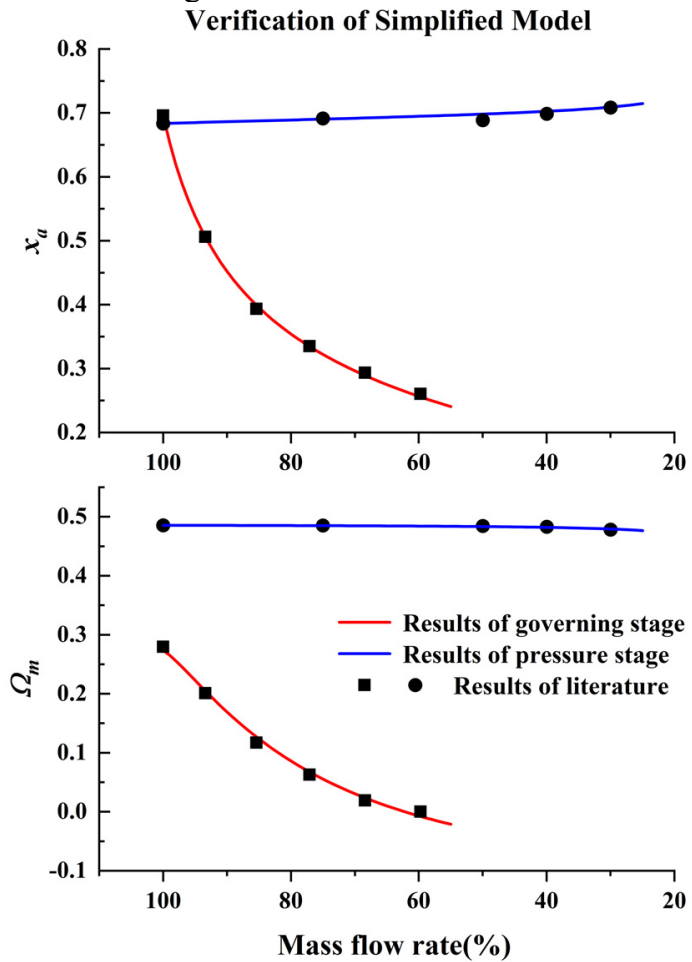

Figure 4. Verification of the simplified model (the top is velocity ratio and the bottom is reaction degree)

As it is shown in Fig. 4, the results calculated by the model are consistent with the reference data when the governing and pressure stages under non-critical conditions. For the assume velocity ratio, the reference fits well with the calculated assume velocity ratio except a large deviation $(1.3 \%$ error) at the $50 \%$ point in the reaction stage. For the reaction degree, the trend is consistent with the results obtained in the literature [7] and also has a small overall error, which is less than $0.5 \%$. Therefore, the simplified model is valid for the calculation of velocity ratio and reaction under offdesign conditions.

\subsection{Results And Discussion of Sensitivity under Off-design Conditions}

The design data of an actual turbine are taken into consideration to study the sensitivity of the governing stage and the low pressure stage under off-design and non-critical conditions. The design parameters, which will be calculated and analysed by the sensitivity model,are shown in Table 1.
By substituting the parameters such as area ratio, the numerical method is able to obtain an accurate value of the reaction degree under off-design conditions.

Table 1. Design parameters of sensitivity analysis.

\begin{tabular}{ccc}
\hline Parameters name & $\begin{array}{c}\text { Governing } \\
\text { stage }\end{array}$ & $\begin{array}{c}\text { Pressure } \\
\text { stage }\end{array}$ \\
\hline $\begin{array}{c}\text { Area ratio } f_{0} \\
\text { Nozzle inlet } \\
\text { angle } \alpha_{1}\left(^{\circ}\right)\end{array}$ & 1.5 & 1.2 \\
$\begin{array}{c}\text { Blade outlet } \\
\text { angle } \beta_{2}\left(^{\circ}\right)\end{array}$ & 15 & 14 \\
$\begin{array}{c}\text { Design reaction } \Omega_{m} \\
\text { Design pressure } \\
\quad \text { ratio } \varepsilon\end{array}$ & 0.168 & 15 \\
$\begin{array}{c}\text { Design velocity } \\
\text { ratio } x_{a}\end{array}$ & 0.67 & 0.500 \\
\hline
\end{tabular}

According to the literature [3] the nozzle factor of the stage is only related to the nozzle configuration and can be considered unchanged. While the blade factor is related to the reaction degree and the exit velocity of the blade, so it is necessary to consider the change in the blade factor due to a large enthalpy drop raise in the governing stage[9]. Also, considering the long-time operation and water conditions, the velocity factors of the stages are changed, and two group of velocity factors are set for the governing stage and pressure stage, one of which is the value of the normal conditions and the other is smaller than the previous one to simulate the situation after long running time. The sensitivity analysis diagrams of the governing and pressure stages are shown in Figures below.

\subsubsection{Sensitivity analysis of governing stage}

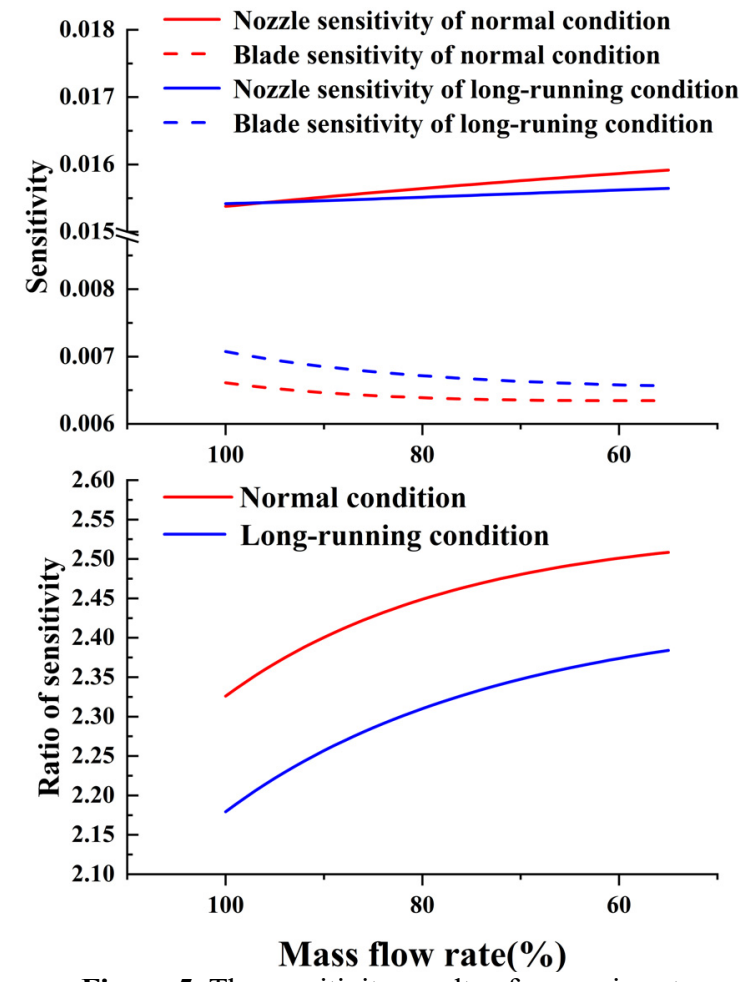

Figure 5. The sensitivity results of governing stage 
From the top part of the Figure.5, it can be depicted that in the process flow rate decline, the nozzle sensitivity increases while the blade sensitivity decreases. This is because the less flow rate of the governing stage will lead to a decrease in the reaction degree, and the stage enthalpy drop gradually rasied, so the enthalpy drop in the nozzle naturally increased, which result in the augument of the nozzle loss propotion. The nozzle sensitivity from 0.0154 at full load condtion to 0.0159 at critical conditions. From the bottom part, due to the variation of two sensitivities, the ratio of them turn to be increased in the whole interval which is about 2.4. The sensitivity of the nozzle and blade and their ratios under long-runing condition are basically the same as the normal case. But the values of the nozzle and blade sensitivities are relatively close to each other in this situation, so the ratio is smaller compared to the normal condition. Based on the sensitivity results, it can be concluded that at any flow condition, increasing the flow efficiency of the nozzle is more helpful to improve the stage perfomance.

\subsubsection{Sensitivity analysis of pressure stage}

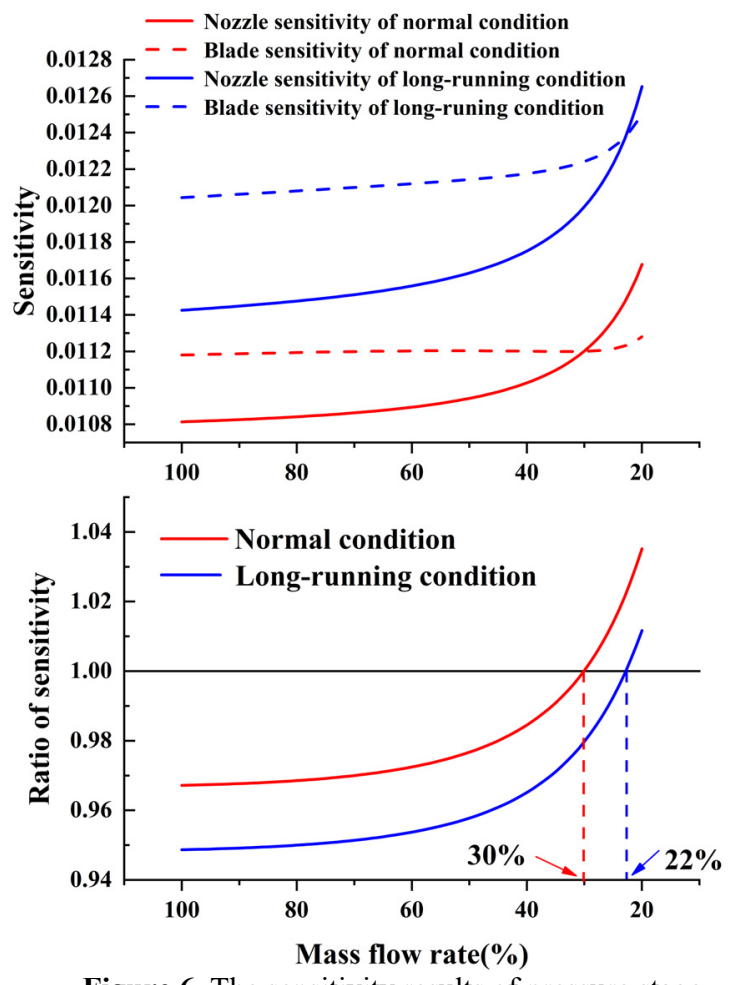

Figure 6. The sensitivity results of pressure stage

From the top panel of Fig. 6, it can be seen that both nozzle sensitivity and blade sensitivity increase with the declining flow rate. This is because when the flow rate decreases, the reaction of the stage will decrease, resulting in an increase in nozzle sensitivity. While the velocity ratio of the stage will raise, which will lead to an increase in the balde sensitivity of the stage according to the analysis in Fig. 3. Long-term running conditions will lead to an augment of nozzle and blade losses in the stage compared to normal conditions. As the reaction degree of the reaction stage is 0.5 , the enthalpy drop in the nozzle and the blade is the same, so the two sensitivities are close in value, and the ratio of the two is around 1 . Though the reaction degree and the velocity ratio change will lead to an increase in the sensitivity of the nozzle, the amplitude in the nozzle is greater than the blade, resulting in the ratio reaching 1 at low flow rates condtions. For normal conditions the critical flow rate is about $30 \%$, while in For long-runing conditions, the critical flow rate is $22 \%$. Therefore, it can be concluded that when the turbine is under high load for a long period of time, improving the velocity factor of the blade is more beneficial to the efficiency of the unit, while when the turbine is under low load, the flow efficiency of the nozzle should be more important.

\section{Conclusion}

(1) In this paper, firstly, the concept of turbine stage velocity factor sensitivity is proposed to quantify the effect of nozzle and blade loss variations on the wheel circumferential efficiency.

(2) The effect of parameters on the sensitivity is analyzed, and the results show that in most cases, the reaction degree is the main factor.

(3) The simplified model to calculate reaction degree and velocity ratio under off-design conditions was established by using Freugel's eqution and the reliability of the result was verified by the reference data.

(4) The sensitivity under off-design conditions is calculated and analyzed. The results show that for the governing stage, the nozzle facor plays a more significant role in improving the stage performance. While for the pressure stage it should be judged according to the flow rate. Specifically speaking,the blade factor is relatively important in a high load and it is reverse in the low load.

\section{References}

1. Wei N. Significance of Loss Models in Aerothermodynamic Simulation for Axial Turbines . KTH. (2000)

2. LI Yong, CHANG Xin-yu. Calculation Method of the Velocity Coefficient and Influent Factor on Nozzle. Turbine Technology. 03 165-167+171. (2015)

3. Kang Song, Yang Jianming, Xu Jianqun . Steam Turbine Theory. (China Electric Power Press, Beijing 2009:16-30.)

4. Shen Shiyi, Zhuang Heqing, Kang Song, Pang Liyun. Steam Turbine Theory. (China Electric Power Press, Beijing 1992.6: 25-37.)

5. Wang Zhongqi, Qin Ren, Theory of Turbine Machine. (China Machine Press, Beijing 1981:32-42)

6. Li Weite, Huang BaoHai. Thermodynamic Calculation of Steam Turbine under Off-design Conditions. (China Electric Power Press, Beijing , 2001.). 
7. Cao Zuqing. Off-design Conditions Characteristics of Steam Turbine. (China Water\&Power Press, Beijing, 1991.)

8. Huang Ru-guang, Yang Jia-ji. Study and Appl ication on Reaction Rule in Stage. Thermal Turbine. 04. $252-254+258$. (2009)

9. Li Huijun, Zhao Xiang. Improvement on Fast Algorithm of Steam Turbine Governing Stages Characteristic Curve. Thermal Power Generation , 10. $94-98 .(2016)$ 